\title{
Strong convergence by a hybrid algorithm for solving generalized mixed equilibrium problems and fixed point problems of a Lipschitz pseudo-contraction in Hilbert spaces
}

\author{
Kasamsuk Ungchittrakool ${ }^{1,2^{*}}$ and Apisit Jarernsuk ${ }^{1}$
}

${ }^{*}$ Correspondence:

kasamsuku@nu.ac.th

${ }^{1}$ Department of Mathematics,

Faculty of Science, Naresuan

University, Phitsanulok, 65000,

Thailand

${ }^{2}$ Centre of Excellence in

Mathematics, CHE, Si Ayutthaya

Road, Bangkok, 10400, Thailand

\section{Springer}

\begin{abstract}
In this paper, we construct a sequence by using some appropriated closed convex sets based on the hybrid shrinking projection methods to find a common solution of fixed point problems of a Lipschitz pseudo-contraction and generalized mixed equilibrium problems in Hilbert spaces. The strong convergence theorems are proved under some mild conditions on scalars. The results not only cover the research work of Yao et al. (Nonlinear Anal. 71:4997-5002, 2009) but can also be applied for finding the common element of the set of zeroes of a Lipschitz monotone mapping and the set of generalized mixed equilibrium problems in Hilbert spaces.
\end{abstract}

MSC: $47 \mathrm{H} 05 ; 47 \mathrm{H} 09 ; 47 \mathrm{H} 10 ; 47 \mathrm{~J} 25$

Keywords: hybrid algorithm; pseudo-contractive mapping; strong convergence; generalized mixed equilibrium problem; Hilbert space

\section{Introduction}

The equilibrium problem theory provides a novel and unified treatment of a wide class of problems which arise in economics, finance, image reconstruction, ecology, transportation, network, elasticity and optimization, and it has been extended and generalized in many directions; see $[1,2]$. In particular, equilibrium problems are related to the problem of finding fixed points problems of some nonlinear mappings. Therefore, it is natural to construct a unified approach to these problems. In this direction, several authors have introduced some iterative schemes for finding a common element of the set of the solutions of equilibrium problems and the set of fixed points (see also [3-7] and the references therein). In this paper, we suggest and analyze a hybrid algorithm for solving generalized mixed equilibrium problems and fixed point problems of a Lipschitz pseudo-contraction in the framework of Hilbert spaces.

Let $E$ be a real Banach space, and $E^{*}$ the dual space of $E$. Let $C$ be a nonempty closed convex subset of $E$. Let $\Theta: C \times C \rightarrow \mathbb{R}$ be a bifunction, $\varphi: C \rightarrow \mathbb{R}$ be a real-valued function, and $A: C \rightarrow E^{*}$ be a nonlinear mapping. The generalized mixed equilibrium problem 
is to find $x \in C$ such that

$$
\Theta(x, y)+\langle A x, y-x\rangle+\varphi(y)-\varphi(x) \geq 0, \quad \forall y \in C .
$$

The solution set of $(1.1)$ is denoted by $\operatorname{GMEP}(\Theta, A, \varphi)$, i.e.,

$$
\operatorname{GMEP}(\Theta, A, \varphi)=\{x \in C: \Theta(x, y)+\langle A x, y-x\rangle+\varphi(y)-\varphi(x) \geq 0, \forall y \in C\}
$$

If $A=0$, the problem (1.1) reduces to the mixed equilibrium problem for $\Theta$, denoted by $\operatorname{MEP}(\Theta, \varphi)$, which is to find $x \in C$ such that

$$
\Theta(x, y)+\varphi(y)-\varphi(x) \geq 0, \quad \forall y \in C
$$

If $\Theta=0$, the problem (1.1) reduces to the mixed variational inequality of Browder type, denoted by $\operatorname{VI}(C, A, \varphi)$, which is to find $x \in C$ such that

$$
\langle A x, y-x\rangle+\varphi(y)-\varphi(x) \geq 0, \quad \forall y \in C
$$

If $A=0$ and $\varphi=0$, the problem (1.1) reduces to the equilibrium problem for $\Theta$ (for short, $E P)$, denoted by $E P(\Theta)$, which is to find $x \in C$ such that

$$
\Theta(x, y) \geq 0, \quad \forall y \in C .
$$

Let $\Theta(x, y)=\langle A x, y-x\rangle$ for all $x, y \in C$. Then $p \in E P(\Theta)$ if and only if $\langle A p, y-p\rangle \geq 0$ for all $y \in C$, i.e., $p$ is a solution of the variational inequality; there are several other problems, for example, the complementarity problem, fixed point problem and optimization problem, which can also be written in the form of an $E P$. In other words, the $E P$ is a unifying model for several problems arising in physics, engineering, science, optimization, economics, etc. Many papers on the existence of solutions of $E P$ have appeared in the literature (see, for example, $[1,8-10]$ and references therein). Motivated by the work [3, 11, 12], Takahashi and Takahashi [4] introduced an iterative scheme by the viscosity approximation method for finding a common element of the set of solutions of the EP (1.2) and the set of fixed points of a nonexpansive mapping in the setting of a Hilbert space. They also studied the strong convergence of the sequences generated by their algorithm for a solution of the $E P$ which is also a fixed point of a nonexpansive mapping defined on a closed convex subset of a Hilbert space.

Recall, a mapping $T$ with domain $D(T)$ and range $R(T)$ in $H$ is called firmly nonexpansive if

$$
\|T x-T y\|^{2} \leq\langle T x-T y, x-y\rangle, \quad \forall x, y \in D(T)
$$

nonexpansive if

$$
\|T x-T y\| \leq\|x-y\|, \quad \forall x, y \in D(T) .
$$


Throughout this paper, $I$ stands for an identity mapping. The mapping $T$ is said to be a strict pseudo-contraction if there exists a constant $0 \leq \kappa<1$ such that

$$
\|T x-T y\|^{2} \leq\|x-y\|^{2}+\kappa\|(I-T) x-(I-T) y\|^{2}, \quad \forall x, y \in D(T) .
$$

In this case, $T$ may be called a $\kappa$-strict pseudo-contraction mapping. In the even that $\kappa=1$, $T$ is said to be a pseudo-contraction, i.e.,

$$
\|T x-T y\|^{2} \leq\|x-y\|^{2}+\|(I-T) x-(I-T) y\|^{2}, \quad \forall x, y \in D(T) .
$$

It is easy to see that (1.3) is equivalent to

$$
\langle x-y,(I-T) x-(I-T) y\rangle \geq 0, \quad \forall x, y \in D(T) .
$$

By definition, it is clear that

$$
\begin{aligned}
\text { firmly nonexpansive } & \Rightarrow \text { nonexpansive } \Rightarrow \text { strict pseudo-contraction } \\
& \Rightarrow \text { pseudo-contraction. }
\end{aligned}
$$

However, the following examples show that the converse is not true.

Example 1.1 (Chidume and Mutangadura [13]) Take $H=\mathbb{R}^{2}, B=\left\{x \in \mathbb{R}^{2}:\|x\| \leq 1\right\}, B_{1}=$ $\left\{x \in B:\|x\| \leq \frac{1}{2}\right\}, B_{2}=\left\{x \in B: \frac{1}{2} \leq\|x\| \leq 1\right\}$. If $x=(a, b) \in H$, we define $x^{\perp}$ to be $(b,-a) \in H$. Define $T: B \rightarrow B$ by

$$
T x= \begin{cases}x+x^{\perp}, & x \in B_{1}, \\ \frac{x}{\|x\|}-x+x^{\perp}, & x \in B_{2} .\end{cases}
$$

Then, $T$ is Lipschitz and a pseudo-contraction but not a strict pseudo-contraction.

Example 1.2 Take $H=\mathbb{R}^{1}$ and define $T: H \rightarrow H$ by $T x=-3 x$. Then, $T$ is a strict pseudocontraction but not a nonexpansive mapping.

Indeed, it is clear that $T$ is not nonexpansive. On the other hand, let us consider

$$
\begin{aligned}
\|T x-T y\|^{2} & =\|(-3 x)-(-3 y)\|^{2}=9\|x-y\|^{2}=\|x-y\|^{2}+8\|x-y\|^{2} \\
& =\|x-y\|^{2}+\frac{16}{2}\|x-y\|^{2}=\|x-y\|^{2}+\frac{1}{2}\|4 x-4 y\|^{2} \\
& =\|x-y\|^{2}+\frac{1}{2}\|(1-(-3)) x-(1-(-3)) y\|^{2} \\
& =\|x-y\|^{2}+\frac{1}{2}\|(I-T) x-(I-T) y\|^{2} \\
& \leq\|x-y\|^{2}+\kappa\|(I-T) x-(I-T) y\|^{2}
\end{aligned}
$$

for all $\kappa \in\left[\frac{1}{2}, 1\right)$. Thus $T$ is a strict pseudo-contraction. 
Example 1.3 Take $H \neq\{0\}$ and let $T=-I$, it is not hard to verify that $T$ is nonexpansive but not firmly nonexpansive.

From a practical point of view, strict pseudo-contractions have more powerful applications than nonexpansive mappings do in solving inverse problems (see [14]). Therefore, it is important to develop a theory of iterative methods for strict pseudo-contractions.

Takahashi and Zembayashi $[5,6]$ proposed some hybrid methods to find the solution of a fixed point problem and an equilibrium problem in Banach spaces. Subsequently, many authors (see, e.g. [15-19] and references therein) have used the hybrid methods to solve fixed point problems and equilibrium problems.

Recently, Yao et al. [20] introduced the hybrid iterative algorithm which just involved one sequence of closed convex set for a pseudo-contractive mapping in Hilbert spaces as follows:

Let $C$ be a nonempty closed convex subset of a real Hilbert space $H$. Let $T: C \rightarrow C$ be a pseudo-contraction. Let $\left\{\alpha_{n}\right\}$ be a sequence in $(0,1)$. Let $x_{0} \in H$. For $C_{1}=C$ and $x_{1}=$ $P_{C_{1}}\left(x_{0}\right)$, define a sequence $\left\{x_{n}\right\}$ of $C$ as follows:

$$
\left\{\begin{array}{l}
y_{n}=\left(1-\alpha_{n}\right) x_{n}+\alpha_{n} T z_{n}, \\
C_{n+1}=\left\{v \in C_{n}:\left\|\alpha_{n}(I-T) y_{n}\right\|^{2} \leq 2 \alpha_{n}\left\langle x_{n}-v,(I-T) y_{n}\right\rangle\right\} \\
x_{n+1}=P_{C_{n+1}}\left(x_{0}\right) .
\end{array}\right.
$$

Theorem 1.4 ([20]) Let C be a nonempty closed convex subset of a real Hilbert space H. Let $T: C \rightarrow C$ be an L-Lipschitz pseudo-contraction such that $F(T) \neq \varnothing$. Assume the sequence $\left\{\alpha_{n}\right\} \subset[a, b]$ for some $a, b \in\left(0, \frac{1}{L+1}\right)$. Then the sequence $\left\{x_{n}\right\}$ generated by (1.4) converges strongly to $P_{F(T)}\left(x_{0}\right)$.

Very recently, Tang et al. [21] generalized the hybrid algorithm (1.4) in the case of the Ishikawa iterative process as follows:

$$
\left\{\begin{aligned}
y_{n}= & \left(1-\alpha_{n}\right) x_{n}+\alpha_{n} T z_{n}, \\
z_{n}= & \left(1-\beta_{n}\right) x_{n}+\beta_{n} T x_{n}, \\
C_{n+1}= & \left\{v \in C_{n}:\left\|\alpha_{n}(I-T) y_{n}\right\|^{2} \leq 2 \alpha_{n}\left\langle x_{n}-v,(I-T) y_{n}\right\rangle\right. \\
& \left.\quad+2 \alpha_{n} \beta_{n} L\left\|x_{n}-T x_{n}\right\|\left\|y_{n}-x_{n}+\alpha_{n}(I-T) y_{n}\right\|\right\}, \\
x_{n+1}= & P_{C_{n+1}}\left(x_{0}\right) .
\end{aligned}\right.
$$

Under some appropriate conditions of $\left\{\alpha_{n}\right\}$ and $\left\{\beta_{n}\right\}$, they proved that (1.5) converges strongly to $P_{F(T)}\left(x_{0}\right)$.

Motivated and inspired by the above research work, in this paper, by employing (1.4) and (1.5), we construct a sequence by using some appropriated closed convex sets based on the hybrid shrinking projection methods to find a common solution of fixed point problems of a Lipschitz pseudo-contraction and generalized mixed equilibrium problems in Hilbert spaces. More precisely, we also provide some applications of the main theorem for finding the common element of the set of zeroes of a Lipschitz monotone mapping and the set of generalized mixed equilibrium problems in Hilbert spaces. 


\section{Preliminaries}

Let $H$ be a real Hilbert space with inner product $\langle\cdot, \cdot\rangle$ and norm $\|\cdot\|$ and let $C$ be a closed convex subset of $H$. For every point $x \in H$, there exists a unique nearest point in $C$, denoted by $P_{C}(x)$, such that

$$
\left\|x-P_{C} x\right\| \leq\|x-y\| \quad \forall y \in C,
$$

where $P_{C}$ is called the metric projection of $H$ onto $C$. We know that $P_{C}$ is a nonexpansive mapping. It is also known that $H$ satisfies Opial's condition, i.e., for any sequence $\left\{x_{n}\right\}$ with $x_{n} \rightarrow x$, the inequality

$$
\liminf _{n \rightarrow \infty}\left\|x_{n}-x\right\|<\liminf _{n \rightarrow \infty}\left\|x_{n}-y\right\|
$$

holds for every $y \in H$ with $y \neq x$.

For a given sequence $\left\{x_{n}\right\} \subset C$, let $\omega_{w}\left(x_{n}\right)=\left\{x: \exists x_{n_{j}} \rightarrow x\right\}$ denote the weak $\omega$-limit set of $\left\{x_{n}\right\}$.

Now we recall some lemmas which will be used in the proof of the main result in the next section. We note that Lemmas 2.1 and 2.2 are well known.

Lemma 2.1 Let $H$ be a real Hilbert space. There holds the following identity

(i) $\|x-y\|^{2}=\|x\|^{2}-\|y\|^{2}-2\langle x-y, y\rangle \forall x, y \in H$.

Lemma 2.2 Let $C$ be a closed convex subset of a real Hilbert space $H$. Given $x \in H$ and $z \in C$. Then $z=P_{C} x$ if and only if there holds the relation

$$
\langle x-z, y-z\rangle \leq 0 \quad \forall y \in C .
$$

For solving the equilibrium problem for a bifunction $\Theta: C \times C \rightarrow \mathbb{R}$, let us assume that $\Theta$ satisfies the following condition:

(A1) $\Theta(x, x)=0$ for all $x \in C$;

(A2) $\Theta$ is monotone, i.e., $\Theta(x, y)+\Theta(y, x) \leq 0$ for all $x, y \in C$;

(A3) for each $x, y, z \in C$,

$$
\lim _{t \downarrow 0} \Theta(t z+(1-t) x, y) \leq \Theta(x, y)
$$

(A4) for each $x \in C, y \longmapsto \Theta(x, y)$ is convex and lower semi-continuous.

For a real Banach space $E$ with norm $\|\cdot\|$, duality product $\langle\cdot, \cdot\rangle$ and dual space $E^{\prime \prime}$, the normalized duality mapping $J: E \rightarrow 2^{E^{n}}$ is defined by

$$
J x=\left\{x^{*} \in E^{*}:\left\langle x, x^{*}\right\rangle=\|x\|^{2}=\left\|x^{*}\right\|^{2}\right\}, \quad \text { for } x \in E .
$$

Lemma 2.3 (Blum and Oettli [1]) Let $C$ be a nonempty closed convex subset of a smooth, strictly convex and reflexive Banach space $E$, and let $\Theta$ be a bifunction of $C \times C$ into $\mathbb{R}$ satisfying (A1)-(A4). Let $r>0$ and $x \in E$. Then, there exists $z \in C$ such that

$$
\Theta(z, y)+\frac{1}{r}\langle y-z, J z-J x\rangle \geq 0, \quad \text { for all } y \in C .
$$


The proof of the following lemma appears in [5, Lemma 2.8].

Lemma 2.4 Let $C$ be a closed convex subset of a uniformly smooth, strictly convex and reflexive Banach space $E$, and let $\Theta$ be a bifunction from $C \times C$ to $\mathbb{R}$ satisfying (A1)-(A4). For $r>0$ and $x \in E$, define a mapping $T_{r}: E \rightarrow C$ as follows:

$$
T_{r} x=\left\{z \in C: \Theta(z, y)+\frac{1}{r}\langle y-z, J z-J x\rangle \geq 0, \text { for all } y \in C\right\}
$$

for all $x \in C$. Then, the following hold:

(i) $T_{r}$ is single-valued;

(ii) $T_{r}$ is firmly nonexpansive-type mapping, i.e., for any $x, y \in H$,

$$
\left\langle T_{r} x-T_{r} y, J T_{r} x-J T_{r} y\right\rangle \leq\left\langle T_{r} x-T_{r} y, J x-J y\right\rangle
$$

(iii) $F\left(T_{r}\right)=E P(\Theta)$;

(iv) $E P(\Theta)$ is closed and convex.

Lemma 2.5 (Zhang [22]) Let $C$ be a closed convex subset of a smooth, strictly convex and reflexive Banach space E. Let $A: C \rightarrow E^{*}$ be a continuous and monotone mapping, $\varphi: C \rightarrow$ $\mathbb{R}$ be a lower semi-continuous and convex function, and $\Theta$ be a bifunction of $C \times C$ to $\mathbb{R}$ satisfying (A1)-(A4). For $r>0$ and $x \in E$. Then, there exists $u \in C$ such that

$$
\Theta(u, y)+\langle A u, y-u\rangle+\varphi(y)-\varphi(u)+\frac{1}{r}\langle y-u, J u-J x\rangle \geq 0, \quad \forall y \in C .
$$

Define a mapping $K_{r}: C \rightarrow C$ as follows:

$$
K_{r}(x)=\left\{u \in C: \Theta(u, y)+\langle A u, y-u\rangle+\varphi(y)-\varphi(u)+\frac{1}{r}\langle y-u, J u-J x\rangle \geq 0, \forall y \in C\right\}
$$

for all $x \in C$. Then, the following conclusions hold:

(i) $K_{r}$ is single-valued;

(ii) $K_{r}$ is firmly nonexpansive-type mapping, i.e., for any $x, y \in E$,

$$
\left\langle K_{r} x-K_{r} y, J K_{r} x-J K_{r} y\right\rangle \leq\left\langle K_{r} x-K_{r} y, J x-J y\right\rangle
$$

(iii) $F\left(K_{r}\right)=G M E P(\Theta, A, \varphi)$;

(iv) $\operatorname{GMEP}(\Theta, A, \varphi)$ is closed and convex;

(v) $\phi\left(p, K_{r} z\right)+\phi\left(K_{r} z, z\right) \leq \phi(p, z), \forall p \in F\left(K_{r}\right), z \in E$.

Remark 2.6 In the framework of a Hilbert space, it is well known that $J=I$ and then $K_{r}$ is firmly nonexpansive.

Lemma 2.7 ([23]) Let $H$ be a real Hilbert space, $C$ a closed convex subset of $H$ and $T$ : $C \rightarrow C$ a continuous pseudo-contractive mapping, then

(i) $F(T)$ is a closed convex subset of $C$.

(ii) $I-T$ is demiclosed at zero, i.e., if $\left\{x_{n}\right\}$ is a sequence in $C$ such that $x_{n} \rightarrow z$ and $(I-T) x_{n} \rightarrow 0$, then $(I-T) z=0$. 
Lemma 2.8 ([24]) Let $C$ be a closed convex subset of $H$. Let $\left\{x_{n}\right\}$ be a sequence in $H$ and $u \in H$. Let $q=P_{C} u$. If $\left\{x_{n}\right\}$ is such that $\omega_{w}\left(x_{n}\right) \subset C$ and satisfies the condition

$$
\left\|x_{n}-u\right\| \leq\|u-q\| \quad \forall n .
$$

Then $x_{n} \rightarrow q$.

Lemma 2.9 Let $\varnothing \neq C \subset H$ be a closed convex set, $a \in \mathbb{R}$ and

$$
K=\{v \in C: a \leq f(v)\}
$$

where $f$ is continuous and concave functional. Then the set $K$ is closed and convex.

Proof It is easy to see that the continuity of $f$ yields the closeness of $K$. Notice that for all $x, y \in K$ and $t \in[0,1]$, we have $t x+(1-t) y \in C, f(x) \geq a, f(y) \geq a$, and then the concavity of $f$ allows

$$
f(t x+(1-t) y) \geq t f(x)+(1-t) f(y) \geq t a+(1-t) a=a .
$$

Thus $K$ is convex.

The following lemma provides some useful properties of a firmly nonexpansive mapping on a Hilbert space.

Lemma 2.10 ([7, Lemma 2.5]) $T$ is firmly nonexpansive if and only if $(I-T)$ is firmly nonexpansive.

\section{Main result}

Theorem 3.1 Let $C$ be a nonempty closed convex subset of a real Hilbert space $H, T: C \rightarrow$ $C$ be an L-Lipschitz pseudo-contraction. Let $\Theta$ be a bifunction from $C \times C$ into $\mathbb{R}$ satisfying (A1)-(A4), $\varphi: C \rightarrow \mathbb{R}$ be a lower semicontinuous and convex function, $A: C \rightarrow H$ be a continuous and monotone mapping such that $\Omega:=F(T) \cap \operatorname{GMEP}(\Theta, A, \varphi) \neq \varnothing$. Let $x_{0} \in H$. For $C_{1}=C$ and $x_{1}=P_{C_{1}}\left(x_{0}\right)$, define a sequence $\left\{x_{n}\right\}$ of $C$ as follows:

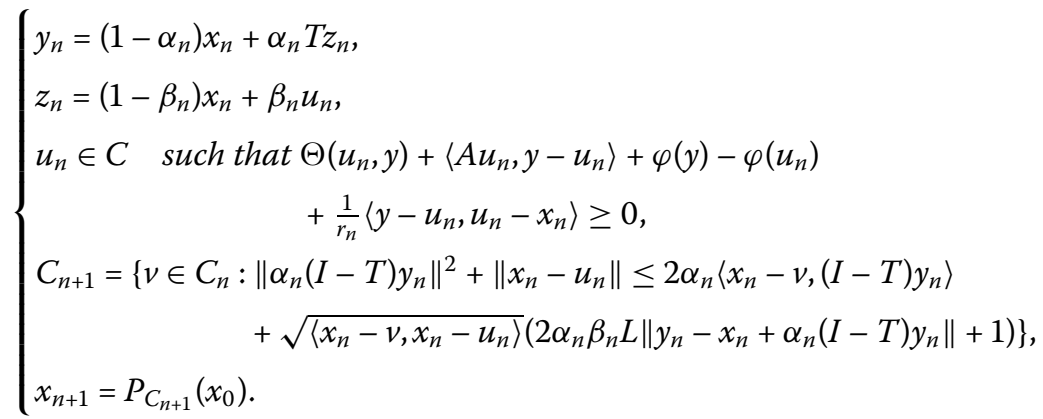

Assume the sequence $\left\{\alpha_{n}\right\},\left\{\beta_{n}\right\}$ and $\left\{r_{n}\right\}$ are such that

(1) $0<a \leq \alpha_{n} \leq b<\frac{1}{L+1}<1$ for all $n \in \mathbb{N}$,

(2) $0 \leq \beta_{n} \leq 1$ for all $n \in \mathbb{N}$, 
(3) $r_{n}>0$ for all $n \in \mathbb{N}$ with $\liminf _{n \rightarrow \infty} r_{n}>0$.

Then $\left\{x_{n}\right\}$ converges strongly to $P_{\Omega}\left(x_{0}\right)$.

Proof By Lemma 2.7(i) and Lemma 2.5(iv), we see that $F(T)$ and $G M E P(\Theta, A, \varphi)$ are closed and convex respectively, then $\Omega$ is also. Hence $P_{\Omega}$ is well defined. Next, we will prove by induction that $\Omega \subset C_{n}$ for all $n \in \mathbb{N}$. Note that $\Omega \subset C=C_{1}$. Assume that $\Omega \subset C_{k}$ holds for some $k \geq 1$. Let $p \in \Omega$, thus $p \in C_{k}$. We observe that

$$
\begin{aligned}
&\left\|x_{k}-p-\alpha_{k}(I-T) y_{k}\right\|^{2} \\
&=\left\|x_{k}-p\right\|^{2}-\left\|\alpha_{k}(I-T) y_{k}\right\|^{2}-2 \alpha_{k}\left\langle(I-T) y_{k}, x_{k}-p-\alpha_{k}(I-T) y_{k}\right\rangle \\
&=\left\|x_{k}-p\right\|^{2}-\left\|\alpha_{k}(I-T) y_{k}\right\|^{2}-2 \alpha_{k}\left\langle(I-T) y_{k}-(I-T) p, y_{k}-p\right\rangle \\
& \quad-2 \alpha_{k}\left\langle(I-T) y_{k}, x_{k}-y_{k}-\alpha_{k}(I-T) y_{k}\right\rangle \\
& \leq\left\|x_{k}-p\right\|^{2}-\left\|\alpha_{k}(I-T) y_{k}\right\|^{2}-2 \alpha_{k}\left\langle(I-T) y_{k}, x_{k}-y_{k}-\alpha_{k}(I-T) y_{k}\right\rangle \\
&=\left\|x_{k}-p\right\|^{2}-\left\|\left(x_{k}-y_{k}\right)+\left(y_{k}-x_{k}+\alpha_{k}(I-T) y_{k}\right)\right\|^{2} \\
& \quad-2 \alpha_{k}\left\langle(I-T) y_{k}, x_{k}-y_{k}-\alpha_{k}(I-T) y_{k}\right\rangle \\
&=\left\|x_{k}-p\right\|^{2}-\left\|x_{k}-y_{k}\right\|^{2}-\left\|y_{k}-x_{k}+\alpha_{k}(I-T) y_{k}\right\|^{2} \\
& \quad-2\left\langle x_{k}-y_{k}, y_{k}-x_{k}+\alpha_{k}(I-T) y_{k}\right\rangle-2 \alpha_{k}\left\langle(I-T) y_{k}, x_{k}-y_{k}-\alpha_{k}(I-T) y_{k}\right\rangle \\
& \leq\left\|x_{k}-p\right\|^{2}-\left\|x_{k}-y_{k}\right\|^{2}-\left\|y_{k}-x_{k}+\alpha_{k}(I-T) y_{k}\right\|^{2} \\
&+2\left|\left\langle x_{k}-y_{k}-\alpha_{k}(I-T) y_{k}, x_{k}-y_{k}-\alpha_{k}(I-T) y_{k}\right\rangle\right| .
\end{aligned}
$$

Consider the last term of (3.2), we obtain

$$
\begin{aligned}
&\left|\left\langle x_{k}-y_{k}-\alpha_{k}(I-T) y_{k}, y_{k}-x_{k}+\alpha_{k}(I-T) y_{k}\right\rangle\right| \\
&=\alpha_{k}\left|\left\langle x_{k}-T z_{k}-(I-T) y_{k}, y_{k}-x_{k}+\alpha_{k}(I-T) y_{k}\right\rangle\right| \\
&=\alpha_{k}\left|\left\langle x_{k}-T x_{k}+T x_{k}-T z_{k}-(I-T) y_{k}, y_{k}-x_{k}+\alpha_{k}(I-T) y_{k}\right\rangle\right| \\
&=\alpha_{k} \mid\left\langle(I-T) x_{k}-(I-T) y_{k}, y_{k}-x_{k}+\alpha_{k}(I-T) y_{k}\right\rangle \\
& \quad+\left\langle T x_{k}-T z_{k}, y_{k}-x_{k}+\alpha_{k}(I-T) y_{k}\right\rangle \mid \\
& \leq \alpha_{k}(L+1)\left\|x_{k}-y_{k}\right\|\left\|y_{k}-x_{k}+\alpha_{k}(I-T) y_{k}\right\|+\alpha_{k} L\left\|x_{k}-z_{k}\right\|\left\|y_{k}-x_{k}+\alpha_{k}(I-T) y_{k}\right\| \\
& \leq \frac{\alpha_{k}(L+1)}{2}\left(\left\|x_{k}-y_{k}\right\|^{2}+\left\|y_{k}-x_{k}+\alpha_{k}(I-T) y_{k}\right\|^{2}\right) \\
&+\alpha_{k} \beta_{k} L\left\|x_{k}-u_{k}\right\|\left\|y_{k}-x_{k}+\alpha_{k}(I-T) y_{k}\right\| .
\end{aligned}
$$

By connecting (3.2) and (3.3), and then by the assumption (1) on $\left\{\alpha_{n}\right\}$, we obtain

$$
\begin{aligned}
\left\|x_{k}-p-\alpha_{k}(I-T) y_{k}\right\|^{2} \leq & \left\|x_{k}-p\right\|^{2}-\left\|x_{k}-y_{k}\right\|^{2}-\left\|y_{k}-x_{k}+\alpha_{k}(I-T) y_{k}\right\|^{2} \\
& +\alpha_{k}(L+1)\left(\left\|x_{k}-y_{k}\right\|^{2}+\left\|y_{k}-x_{k}+\alpha_{k}(I-T) y_{k}\right\|^{2}\right) \\
& +2 \alpha_{k} \beta_{k} L\left\|x_{k}-u_{k}\right\|\left\|y_{k}-x_{k}+\alpha_{k}(I-T) y_{k}\right\| \\
\leq & \left\|x_{k}-p\right\|^{2}+2 \alpha_{k} \beta_{k} L\left\|x_{k}-u_{k}\right\|\left\|y_{k}-x_{k}+\alpha_{k}(I-T) y_{k}\right\| .
\end{aligned}
$$


Notice that $u_{k}=K_{r_{k}} x_{k}$ and by Lemma 2.10, we observe that

$$
\begin{aligned}
\left\|x_{k}-u_{k}\right\|^{2} & =\left\|\left(I-K_{r_{k}}\right) x_{k}-\left(I-K_{r_{k}}\right) p\right\|^{2} \\
& \leq\left\langle\left(I-K_{r_{k}}\right) x_{k}-\left(I-K_{r_{k}}\right) p, x_{k}-p\right\rangle \\
& =\left\langle\left(I-K_{r_{k}}\right) x_{k}, x_{k}-p\right\rangle .
\end{aligned}
$$

So, we have

$$
\left\|x_{k}-u_{k}\right\| \leq \sqrt{\left\langle x_{k}-p, x_{k}-u_{k}\right\rangle}
$$

Joining (3.4) and (3.5), we obtain

$$
\begin{aligned}
& \left\|x_{k}-p-\alpha_{k}(I-T) y_{k}\right\|^{2} \\
& \quad \leq\left\|x_{k}-p\right\|^{2}+2 \alpha_{k} \beta_{k} L \sqrt{\left\langle x_{k}-p, x_{k}-u_{k}\right\rangle}\left\|y_{k}-x_{k}+\alpha_{k}(I-T) y_{k}\right\| .
\end{aligned}
$$

Notice that

$$
\left\|x_{k}-p-\alpha_{k}(I-T) y_{k}\right\|^{2}=\left\|x_{k}-p\right\|^{2}-2 \alpha_{k}\left\langle x_{k}-p,(I-T) y_{k}\right\rangle+\left\|\alpha_{k}(I-T) y_{k}\right\|^{2} \text {. }
$$

By (3.6) and (3.7), we have

$$
\begin{aligned}
& \left\|\alpha_{k}(I-T) y_{k}\right\|^{2} \\
& \quad \leq 2 \alpha_{k}\left\langle x_{k}-p,(I-T) y_{k}\right\rangle+2 \alpha_{k} \beta_{k} L \sqrt{\left\langle x_{k}-p, x_{k}-u_{k}\right\rangle}\left\|y_{k}-x_{k}+\alpha_{k}(I-T) y_{k}\right\| .
\end{aligned}
$$

Combining (3.8) and (3.5), we obtain

$$
\begin{aligned}
& \left\|\alpha_{k}(I-T) y_{k}\right\|^{2}+\left\|x_{k}-u_{k}\right\| \\
& \quad \leq 2 \alpha_{k}\left\langle x_{k}-p,(I-T) y_{k}\right\rangle+\sqrt{\left\langle x_{k}-p, x_{k}-u_{k}\right\rangle}\left(2 \alpha_{k} \beta_{k} L\left\|y_{k}-x_{k}+\alpha_{k}(I-T) y_{k}\right\|+1\right) .
\end{aligned}
$$

Therefore, $p \in C_{k+1}$. By mathematical induction, we have $\Omega \subset C_{n}$ for all $n \in \mathbb{N}$.

Let $f_{n}(\cdot):=2 \alpha_{n}\left\langle x_{n}-(\cdot),(I-T) y_{n}\right\rangle+\sqrt{\left\langle x_{n}-(\cdot), x_{n}-u_{n}\right\rangle}\left(2 \alpha_{n} \beta_{n} L\left\|y_{n}-x_{n}+\alpha_{n}(I-T) y_{n}\right\|+1\right)$, it is not hard to see that the linearity of $\left\langle x_{n}-(\cdot),(I-T) y_{n}\right\rangle$ and $\left\langle x_{n}-(\cdot), x_{n}-u_{n}\right\rangle$ together with the continuity and concavity of $\sqrt{(\cdot)}$ allow $f_{n}$ to be continuous and concave. By Lemma 2.9, $C_{n}$ is closed and convex for all $n \in \mathbb{N}$. Therefore, $\left\{x_{n}\right\}$ is well defined. From $x_{n}=P_{C_{n}}\left(x_{0}\right)$, we have $\left\langle x_{0}-x_{n}, x_{n}-y\right\rangle \geq 0$ for all $y \in C_{n}$. Using $\Omega \subset C_{n}$, we also have $\left\langle x_{0}-x_{n}, x_{n}-u\right\rangle \geq 0$ for all $u \in \Omega$. So, for $u \in \Omega$, we have

$$
\begin{aligned}
0 & \leq\left\langle x_{0}-x_{n}, x_{n}-u\right\rangle=\left\langle x_{0}-x_{n}, x_{n}-x_{0}+x_{0}-u\right\rangle \\
& =-\left\|x_{0}-x_{n}\right\|^{2}+\left\langle x_{0}-x_{n}, x_{0}-u\right\rangle \\
& \leq-\left\|x_{0}-x_{n}\right\|^{2}+\left\|x_{0}-x_{n}\right\|\left\|x_{0}-u\right\| .
\end{aligned}
$$

Hence,

$$
\left\|x_{0}-x_{n}\right\| \leq\left\|x_{0}-u\right\| \quad \text { for all } u \in \Omega
$$


This implies that $\left\{x_{n}\right\}$ is bounded and then $\left\{y_{n}\right\},\left\{T y_{n}\right\}$ and $\left\{u_{n}\right\}$ are bounded too.

From $x_{n}=P_{C_{n}}\left(x_{0}\right)$ and $x_{n+1}=P_{C_{n+1}}\left(x_{0}\right) \in C_{n+1} \subset C_{n}$, we have

$$
\left\langle x_{0}-x_{n}, x_{n}-x_{n+1}\right\rangle \geq 0 \text {. }
$$

Hence,

$$
\begin{aligned}
0 & \leq\left\langle x_{0}-x_{n}, x_{n}-x_{n+1}\right\rangle=\left\langle x_{0}-x_{n}, x_{n}-x_{0}+x_{0}-x_{n+1}\right\rangle \\
& =-\left\|x_{0}-x_{n}\right\|^{2}+\left\langle x_{0}-x_{n}, x_{0}-x_{n+1}\right\rangle \\
& \leq-\left\|x_{0}-x_{n}\right\|^{2}+\left\|x_{0}-x_{n}\right\|\left\|x_{0}-x_{n+1}\right\|,
\end{aligned}
$$

and therefore

$$
\left\|x_{0}-x_{n}\right\| \leq\left\|x_{0}-x_{n+1}\right\|
$$

which implies that $\lim _{n \rightarrow \infty}\left\|x_{n}-x_{0}\right\|$ exists. From Lemma 2.1 and (3.10), we obtain

$$
\begin{aligned}
\left\|x_{n+1}-x_{n}\right\|^{2} & =\left\|\left(x_{n+1}-x_{0}\right)-\left(x_{n}-x_{0}\right)\right\|^{2} \\
& =\left\|x_{n+1}-x_{0}\right\|^{2}-\left\|x_{n}-x_{0}\right\|^{2}-2\left\langle x_{n+1}-x_{n}, x_{n}-x_{0}\right\rangle \\
& \leq\left\|x_{n+1}-x_{0}\right\|^{2}-\left\|x_{n}-x_{0}\right\|^{2} \rightarrow 0 \quad \text { as } n \rightarrow \infty .
\end{aligned}
$$

Since $x_{n+1} \in C_{n+1} \subset C_{n}$, we have

$$
\begin{aligned}
& \left\|\alpha_{n}(I-T) y_{n}\right\|^{2}+\left\|x_{n}-u_{n}\right\| \\
& \leq 2 \alpha_{n}\left\langle x_{n}-x_{n+1},(I-T) y_{n}\right\rangle \\
& \quad+\sqrt{\left\langle x_{n}-x_{n+1}, x_{n}-u_{n}\right\rangle}\left(2 \alpha_{n} \beta_{n} L\left\|y_{n}-x_{n}+\alpha_{n}(I-T) y_{n}\right\|+1\right) \\
& \rightarrow 0 \quad \text { as } n \rightarrow \infty .
\end{aligned}
$$

Therefore, we obtain

$$
\left\|y_{n}-T y_{n}\right\| \rightarrow 0 \text { and } \quad\left\|x_{n}-u_{n}\right\| \rightarrow 0 \quad \text { as } n \rightarrow \infty \text {. }
$$

We note that

$$
\begin{aligned}
\left\|x_{n}-T x_{n}\right\| & \leq\left\|x_{n}-y_{n}\right\|+\left\|y_{n}-T y_{n}\right\|+\left\|T y_{n}-T x_{n}\right\| \\
& \leq(L+1)\left\|x_{n}-y_{n}\right\|+\left\|y_{n}-T y_{n}\right\| \\
& \leq \alpha_{n}(L+1)\left\|x_{n}-T z_{n}\right\|+\left\|y_{n}-T y_{n}\right\| \\
& \leq \alpha_{n}(L+1)\left\|x_{n}-T x_{n}\right\|+\alpha_{n}(L+1)\left\|T x_{n}-T z_{n}\right\|+\left\|y_{n}-T y_{n}\right\| \\
& \leq \alpha_{n}(L+1)\left\|x_{n}-T x_{n}\right\|+\alpha_{n} \beta_{n} L(L+1)\left\|x_{n}-u_{n}\right\|+\left\|y_{n}-T y_{n}\right\|,
\end{aligned}
$$

that is,

$$
\left\|x_{n}-T x_{n}\right\| \leq \frac{\alpha_{n} \beta_{n} L(L+1)}{1-\alpha_{n}(L+1)}\left\|x_{n}-u_{n}\right\|+\frac{1}{1-\alpha_{n}(L+1)}\left\|y_{n}-T y_{n}\right\| \rightarrow 0 \quad \text { as } n \rightarrow \infty
$$


Next, we will show that

$$
\omega_{w}\left(x_{n}\right) \subset \Omega \text {. }
$$

Since $\left\{x_{n}\right\}$ is bounded, the reflexivity of $H$ guarantees that $\omega_{w}\left(x_{n}\right) \neq \varnothing$. Let $p \in \omega_{w}\left(x_{n}\right)$, then there exists a subsequence $\left\{x_{n_{i}}\right\}$ of $\left\{x_{n}\right\}$ such that $x_{n_{i}} \rightarrow p$ and by Lemma 2.7(ii), we have $p \in F(T)$. On the other hand, since $\left\|x_{n}-u_{n}\right\| \rightarrow 0$ and $x_{n_{i}} \rightarrow p$, we have $u_{n_{i}} \rightarrow p$. Define $G: C \times C \rightarrow \mathbb{R}$ by $G(x, y)=\Theta(x, y)+\langle A x, y-x\rangle+\varphi(y)-\varphi(x)$ for all $x, y \in C$. It is not hard to verify that $G$ satisfies conditions (A1)-(A4). It follows from $u_{n}=K_{r_{n}} x_{n}$ and (A2) that

$$
\frac{1}{r_{n}}\left\langle y-u_{n}, u_{n}-x_{n}\right\rangle \geq G\left(y, u_{n}\right) \quad \text { for all } y \in C .
$$

Replacing $n$ by $n_{i}$, we have

$$
\left\langle y-u_{n_{i}}, \frac{u_{n_{i}}-x_{n_{i}}}{r_{n_{i}}}\right\rangle \geq G\left(y, u_{n_{i}}\right)
$$

By using (A4) and the assumption (3) on $\left\{r_{n}\right\}$, we obtain $0 \geq G(y, p)$ for all $y \in C$. For $t \in(0,1]$ and $y \in C$, let $y_{t}=t y+(1-t) p$. So, from (A1) and (A4) we have

$$
0=G\left(y_{t}, y_{t}\right)=G\left(y_{t}, t y+(1-t) p\right) \leq t G\left(y_{t}, y\right)+(1-t) G\left(y_{t}, p\right) \leq t G\left(y_{t}, y\right) .
$$

Dividing by $t$, we have

$$
G\left(y_{t}, y\right) \geq 0 \quad \text { for all } y \in C \text {. }
$$

From (A3) we have $0 \leq \lim _{t \rightarrow 0} G\left(y_{t}, y\right)=\lim _{t \rightarrow 0} G(t y+(1-t) p, y) \leq G(p, y)$ for all $y \in C$, and hence $p \in \operatorname{GMEP}(\Theta, A, \varphi)$. So, $p \in F(T) \cap \operatorname{GMEP}(\Theta, A, \varphi)=\Omega$ and then we have (3.11). Therefore, by inequality (3.9) and Lemma 2.8 , we obtain $\left\{x_{n}\right\}$ converges strongly to $P_{\Omega}\left(x_{0}\right)$. This completes the proof.

Remark 3.2 It is interesting that the assumption on a sequence of scalars $\left\{\beta_{n}\right\}$ is a very mild condition. This is a direct result of the firmly nonexpansiveness of $I-K_{r_{n}}$ together with the structure and the definition of the set $C_{n}$. If $\beta_{n}=0$ for all $n$, then $z_{n}=x_{n}$ and the sequence $\left\{y_{n}\right\}$ and $\left\{u_{n}\right\}$ are independent. However, the properties of $C_{n}$ still force to produce the sequence $\left\{x_{n}\right\}$ to cause a convergence to the common solution $P_{\Omega}\left(x_{0}\right)$.

If $A=0$ and $\varphi=0$, then we have the following corollary.

Corollary 3.3 Let C be a nonempty closed convex subset of a real Hilbert space $H, T: C \rightarrow$ $C$ be an L-Lipschitz pseudo-contraction. Let $\Theta$ be a bifunction from $C \times C$ into $\mathbb{R}$ satisfying (A1)-(A4), such that $\Omega:=F(T) \cap E P(\Theta) \neq \varnothing$. Let $x_{0} \in H$. For $C_{1}=C$ and $x_{1}=P_{C_{1}}\left(x_{0}\right)$, define 
a sequence $\left\{x_{n}\right\}$ of $C$ as follows:

$$
\left\{\begin{array}{l}
y_{n}=\left(1-\alpha_{n}\right) x_{n}+\alpha_{n} T z_{n}, \\
z_{n}=\left(1-\beta_{n}\right) x_{n}+\beta_{n} u_{n}, \\
\begin{array}{l}
u_{n} \in C \quad \text { such that } \Theta\left(u_{n}, y\right)+\frac{1}{r_{n}}\left\langle y-u_{n}, u_{n}-x_{n}\right\rangle \geq 0, \\
C_{n+1}=\left\{v \in C_{n}:\left\|\alpha_{n}(I-T) y_{n}\right\|^{2}+\left\|x_{n}-u_{n}\right\| \leq 2 \alpha_{n}\left\langle x_{n}-v,(I-T) y_{n}\right\rangle\right. \\
\left.\quad+\sqrt{\left\langle x_{n}-v, x_{n}-u_{n}\right\rangle}\left(2 \alpha_{n} \beta_{n} L\left\|y_{n}-x_{n}+\alpha_{n}(I-T) y_{n}\right\|+1\right)\right\}
\end{array} \\
\begin{array}{rl}
x_{n+1}= & P_{C_{n+1}}\left(x_{0}\right) .
\end{array}
\end{array}\right.
$$

Assume the sequence $\left\{\alpha_{n}\right\},\left\{\beta_{n}\right\}$ and $\left\{r_{n}\right\}$ are as in Theorem 3.1. Then $\left\{x_{n}\right\}$ converges strongly to $P_{\Omega}\left(x_{0}\right)$.

Corollary 3.4 (Yao et al. [20, Theorem 3.1]) Let $C$ be a nonempty closed convex subset of a real Hilbert space H. Let $T: C \rightarrow C$ be an L-Lipschitz pseudo-contraction such that $F(T) \neq \varnothing$. Assume that $\left\{\alpha_{n}\right\}$ is a sequence such that $0<a \leq \alpha_{n} \leq b<\frac{1}{L+1}<1$ for all $n$. Then the sequence $\left\{x_{n}\right\}$ generated by (1.4) converges strongly to $P_{F(T)}\left(x_{0}\right)$.

Proof Put $\Theta=0, A=0, \varphi=0$ and $r_{n}=1$ for all $n \geq 1$ in Theorem 3.1. Then, $K_{r_{n}}=P_{C}$ for all $n \geq 1$. So, $u_{n}=P_{C} x_{n}$ for all $n \geq 1$ (note that $x_{1}=P_{C} x_{0}$ ). Since $x_{n}=P_{C_{n}} x_{0} \in C_{n} \subset C$ for all $n \geq 1$, so we have $u_{n}=x_{n}$ and then $z_{n}=x_{n}$ for all $n \geq 1$. Thus $x_{n}-u_{n}=0$ for all $n \geq 1$. For this reason, (1.4) is a special case of (3.1). Applying Theorem 3.1, we have the desired result.

Recall that a mapping $B$ is said to be monotone, if $\langle x-y, B x-B y\rangle \geq 0$ for all $x, y \in H$ and inverse strongly monotone if there exists a real number $\gamma>0$ such that $\langle x-y, B x-B y\rangle \geq$ $\gamma\|B x-B y\|^{2}$ for all $x, y \in H$. For the second case, $B$ is said to be $\gamma$-inverse strongly monotone. It follows immediately that if $B$ is $\gamma$-inverse strongly monotone, then $B$ is monotone and Lipschitz continuous, that is, $\|B x-B y\| \leq \frac{1}{\gamma}\|x-y\|$. The pseudo-contractive mapping and strictly pseudo-contractive mapping are strongly related to the monotone mapping and inverse strongly monotone mapping, respectively. It is well known that

(i) $B$ is monotone $\Longleftrightarrow T:=(I-B)$ is pseudo-contractive.

(ii) $B$ is inverse strongly monotone $\Longleftrightarrow T:=(I-B)$ is strictly pseudo-contractive.

Indeed, for (ii), we notice that the following equality always holds in a real Hilbert space:

$$
\|(I-B) x-(I-B) y\|^{2}=\|x-y\|^{2}+\|B x-B y\|^{2}-2\langle x-y, B x-B y\rangle \quad \forall x, y \in H,
$$

without loss of generality, we can assume that $\gamma \in\left(0, \frac{1}{2}\right]$, and then it yields

$$
\begin{aligned}
&\langle x-y, B x-B y\rangle \geq \gamma\|B x-B y\|^{2} \\
& \Longleftrightarrow \quad-2\langle x-y, B x-B y\rangle \leq-2 \gamma\|B x-B y\|^{2} \\
& \Longleftrightarrow \quad\|(I-B) x-(I-B) y\|^{2} \leq\|x-y\|^{2}+(1-2 \gamma)\|B x-B y\|^{2} \quad(v i a(3.12)) \\
& \Longleftrightarrow \quad\|T x-T y\|^{2} \leq\|x-y\|^{2}+\kappa\|(I-T) x-(I-T) y\|^{2}
\end{aligned}
$$

(where $T:=(I-B)$ and $\kappa:=1-2 \gamma$ ). 
Corollary 3.5 Let $C, H, \Theta, A$ and $\varphi$ be as in Theorem 3.1 and let $B: H \rightarrow H$ be an $L$ Lipschitz monotone mapping such that $\Omega=B^{-1}(0) \cap \operatorname{GMEP}(\Theta, A, \varphi) \neq \varnothing$. Let $x_{0} \in H$. For $C_{1}=C$ and $x_{1}=P_{C_{1}}\left(x_{0}\right)$, define a sequence $\left\{x_{n}\right\}$ of $C$ as follows:

$$
\left\{\begin{array}{l}
y_{n}=x_{n}-\alpha_{n}\left(x_{n}-z_{n}\right)-\alpha_{n} B z_{n}, \\
z_{n}=\left(1-\beta_{n}\right) x_{n}+\beta_{n} u_{n}, \\
\begin{array}{r}
u_{n} \in C \quad \text { such that } \Theta\left(u_{n}, y\right)+\left\langle A u_{n}, y-u_{n}\right\rangle+\varphi(y)-\varphi\left(u_{n}\right) \\
\quad+\frac{1}{r_{n}}\left\langle y-u_{n}, u_{n}-x_{n}\right\rangle \geq 0,
\end{array} \\
\begin{array}{c}
C_{n+1}=\left\{v \in C_{n}:\right. \\
\quad\left\|\alpha_{n} B y_{n}\right\|^{2}+\left\|x_{n}-u_{n}\right\| \leq 2 \alpha_{n}\left\langle x_{n}-v, B y_{n}\right\rangle \\
\left.\quad+\sqrt{\left\langle x_{n}-v, x_{n}-u_{n}\right\rangle}\left(2 \alpha_{n} \beta_{n} L\left\|y_{n}-x_{n}+\alpha_{n} B y_{n}\right\|+1\right)\right\}
\end{array} \\
x_{n+1}=P_{C_{n+1}}\left(x_{0}\right) .
\end{array}\right.
$$

Assume $0<a \leq \alpha_{n} \leq b<\frac{1}{L+2}<1$ for all $n \in \mathbb{N},\left\{\beta_{n}\right\}$ and $\left\{r_{n}\right\}$ are as in Theorem 3.1. Then $\left\{x_{n}\right\}$ converges strongly to $P_{\Omega}\left(x_{0}\right)$.

Proof Let $T:=(I-B)$. Then $T$ is pseudo-contractive and $(L+2)$-Lipschitz. Hence, it follows from Theorem 3.1, we have the desired result.

\section{Competing interests}

The authors declare that they have no competing interests.

\section{Authors' contributions}

All authors read and approved the final manuscript.

\section{Acknowledgements}

The authors would like to thank the Centre of Excellence in Mathematics under the Commission on Higher Education, Ministry of Education, Thailand. They also thank the Editor and two anonymous referees for reading this paper carefully and providing valuable comments to improve the original version of this paper. The project was supported by Centre of Excellence in Mathematics, CHE, Si Ayutthaya Road, Bangkok, 10400, Thailand.

\section{Received: 18 February 2012 Accepted: 29 August 2012 Published: 12 September 2012}

\section{References}

1. Blum, E, Oettli, W: From optimization and variational inequalities to equilibrium problem. Math. Stud. 63(1), 123-145 (1994)

2. Noor, MA, Oettli, W: On general nonlinear complementarity problems and quasi equilibria. Matematiche 49, 313-331 (1994)

3. Combettes, PL, Hirstoaga, SA: Equilibrium programming in Hilbert spaces. J. Nonlinear Convex Anal. 6, 117-136 (2005)

4. Takahashi, S, Takahashi, W: Viscosity approximation methods for equilibrium problems and fixed point problems in Hilbert spaces. J. Math. Anal. Appl. 331, 506-515 (2007)

5. Takahashi, W, Zembayashi, K: Strong and weak convergence theorems for equilibrium problems and relatively nonexpansive mappings in Banach spaces. Nonlinear Anal. 70, 45-57 (2009)

6. Takahashi, W, Zembayashi, K: Strong convergence theorem by a new hybrid method for equilibrium problems and relatively nonexpansive mappings. Fixed Point Theory Appl. 2008, Article ID 528476 (2008)

7. Jarernsuk, A, Ungchittrakool, K: Strong convergence by a hybrid algorithm for solving equilibrium problem and fixed point problem of a Lipschitz pseudo-contraction in Hilbert spaces. Thai J. Math. 10(1), 181-194 (2012)

8. Flores-Bazan, F: Existence theory for finite-dimensional pseudomonotone equilibrium problems. Acta Appl. Math. 77, 249-297 (2003)

9. Hadjisavvas, N, Komlósi, S, Schaible, S: Handbook of Generalized Convexity and Generalized Monotonicity. Springer, New York (2005)

10. Hadjisavvas, N, Schaible, S: From scalar to vector equilibrium problems in the quasimonotone case. J. Optim. Theory Appl. 96, 297-309 (1998)

11. Moudafi, A: Viscosity approximation methods for fixed-point problems. J. Math. Anal. Appl. 241, 46-55 (2000)

12. Tada, A, Takahashi, W: Strong convergence theorem for an equilibrium problem and a nonexpansive mapping. In: Takahashi, W, Tanaka, T (eds.) Nonlinear Analysis and Convex Analysis, pp. 609-617. Yokohama Publishers, Yokohama (2006)

13. Chidume, CE, Mutangadura, SA: An example on the Mann iteration method for Lipschitz pseudocontractions. Proc Am. Math. Soc. 129(8), 2359-2363 (2001) 
14. Scherzer, O: Convergence criteria of iterative methods based on Landweber iteration for solving nonlinear problems. J. Math. Anal. Appl. 194, 911-933 (1991)

15. Kumam, P, Petrot, N, Wangkeeree, R: A hybrid iterative scheme for equilibrium problems and fixed point problems of asymptotically k-strict pseudo-contractions. J. Comput. Appl. Math. 233(8), 2013-2026 (2010)

16. Kumam, $\mathrm{P}$, Wattanawitoon, $\mathrm{K}$ : Convergence theorems of a hybrid algorithm for equilibrium problems. Nonlinear Anal. Hybrid Syst. 3, 386-394 (2009)

17. Qin, XL, Zhou, HY, Kang, SM: Convergence theorems of common elements for equilibrium problems and fixed point problems in Banach spaces. J. Comput. Appl. Math. 225(1), 20-30 (2009)

18. Plubtieng, S, Ungchittrakool, K: Approximation of common fixed points for a countable family of relatively nonexpansive mappings in a Banach space and applications. Nonlinear Anal. 72(6), 2896-2908 (2010)

19. Ungchittrakool, K: A strong convergence theorem for a common fixed point of two sequences of strictly pseudo contractive mappings in Hilbert spaces and applications. Abstr. Appl. Anal. 2010, Article ID 876819 (2010)

20. Yao, Y, Liou, Y-C, Marino, G: A hybrid algorithm for pseudo-contractive mappings. Nonlinear Anal. 71, 4997-5002 (2009)

21. Tang, Y-C, Peng, J-G, Liu, L-W: Strong convergence theorem for pseudo-contractive mappings in Hilbert spaces. Nonlinear Anal. 74(2), 380-385 (2011)

22. Zhang, S: Generalized mixed equilibrium problem in Banach spaces. Appl. Math. Mech. 30, 1105-1112 (2009)

23. Zhang, QB, Cheng, CZ: Strong convergence theorem for a family of Lipschitz pseudocontractive mappings in a Hilbert space. Math. Comput. Model. 48, 480-485 (2008)

24. Matinez-Yanes, C, Xu, HK: Strong convergence of the CQ method for fixed point process. Nonlinear Anal. 64, 2400-2411 (2006)

doi:10.1186/1687-1812-2012-147

Cite this article as: Ungchittrakool and Jarernsuk: Strong convergence by a hybrid algorithm for solving generalized mixed equilibrium problems and fixed point problems of a Lipschitz pseudo-contraction in Hilbert spaces. Fixed Point Theory and Applications 2012 2012:147.

\section{Submit your manuscript to a SpringerOpen ${ }^{\odot}$ journal and benefit from:}

- Convenient online submission

- Rigorous peer review

- Immediate publication on acceptance

- Open access: articles freely available online

- High visibility within the field

- Retaining the copyright to your article 